\title{
Le filiere agroalimentari al tempo del COVID-19 in Europa e in Italia. Una sfida tra food security, ripresa e dinamiche dei mercati, tutela del lavoro e dei consumatori, Green Deal, incertezze climatiche
}

\section{L'impatto della pandemia sulle filiere agroalimentari}

Tra le poche attività ammesse durante il lockdown alcune di quelle svolte dalle filiere agroalimentari sono state sempre operative. Non poteva che essere così, stante l'ineludibile funzione vitale assolta dal cibo e l'obbligo delle Istituzioni europee e nazionali a renderne possibile l'approvvigionamento attraverso modalità improntate ai principi di responsabilità e solidarietà.

Tuttavia, la forte contrazione economica avviata dall'emergenza sanitaria non ha lasciato immune il comparto durante il suo irrompere, lungo il volgere della sua persistenza e il cammino verso la ripresa.

Diversi i contraccolpi sia per le filiere risultate più idonee a fronteggiare la contingenza sia per le altre meno adatte e, in quanto tali, rimaste ferme anche in mancanza di impedimenti legali.

Ecco, al riguardo, qualche risultato emerso dal "monitoraggio" effettuato per svolgere queste riflessioni.

\footnotetext{
* Università degli Studi di Udine, Italia.
} 
La produzione primaria di frutta e verdura, se non ha subito la scarsità di domanda da parte dei mercati, non sempre riesce a dare risposta a causa delle limitazioni al flusso di lavoratori stranieri che da diversi anni si occupano, in misura consistente, della raccolta dei prodotti sui campi di alcuni Paesi europei, tra cui l'Italia ove la circostanza epidemica ha riportato l'attenzione dialettica sulla piaga del caporalato implementando il fenomeno "storico"1 con quello dei riders del food delivery.

Nonostante l'enfasi diffusamente posta sulla continuità produttiva del settore primario e sull'importante ruolo svolto anche dalle attività ad esso collegate per assicurare le forniture di cibo ai consumatori, nell'anno 2020 e in buona parte del 2021 la pandemia ha mandato in debacle pure il comparto agroalimentare, caratterizzato da una molteplicità di settori produttivi e di mercati.

Gravi danni economici hanno colpito e continuano ad affliggere le imprese che realizzano beni di fascia alta soprattutto da esportazione e ristorazione, come carni, formaggi, alcuni tipi di salumi, vini, prodotti biologici, e ciò, nonostante la ricettività della Grande distribuzione organizzata e l'aumento dell'e-commerce.

Le misure di distanziamento sociale hanno favorito i consumi domestici di cereali e loro derivati, conserve, surgelati, prosciutti e hanno messo in crisi le attività di florovivaismo, agriturismo, enoturismo, street food.

${ }^{1}$ Con il termine caporalato si indica il reato che, ai sensi dell'art. 630-bis del codice penale italiano sul reato di intermediazione illecita e sfruttamento del lavoro, è sanzionato quale delitto e comporta la reclusione, oltre all'elevazione della multa, per chiunque: "1) recluta manodopera allo scopo di destinarla al lavoro presso terzi in condizioni di sfruttamento, approfittando dello stato di bisogno dei lavoratori; 2) utilizza, assume o impiega manodopera, anche mediante l'attività di intermediazione di cui al numero 1), sottoponendo i lavoratori a condizioni di sfruttamento ed approfittando del loro stato di bisogno". "Ai fini del medesimo articolo, costituisce indice di sfruttamento la sussistenza di una o più delle seguenti condizioni: 1) la reiterata corresponsione di retribuzioni in modo palesemente difforme dai contratti collettivi nazionali o territoriali stipulati dalle organizzazioni sindacali più rappresentative a livello nazionale, o comunque sproporzionato rispetto alla quantità e qualità del lavoro prestato; 2) la reiterata violazione della normativa relativa all'orario di lavoro, ai periodi di riposo, al riposo settimanale, all'aspettativa obbligatoria, alle ferie; 3) la sussistenza di violazioni delle norme in materia di sicurezza e igiene nei luoghi di lavoro; 4) la sottoposizione del lavoratore a condizioni di lavoro, a metodi di sorveglianza o a situazioni alloggiative degradanti"'. Si evidenzia come l'attuale testo dell'articolo derivi dalla modifica apportata dalla 1. 29 ottobre 2016, n. 199 recante "Disposizioni in materia di contrasto ai fenomeni del lavoro nero, dello sfruttamento del lavoro in agricoltura e di riallineamento retributivo nel settore agricolo". Recentemente in giurisprudenza è stato ritenuto non necessario, ai fini della qualificazione del reato di caporalato, che sussistano contemporaneamente tutti gli elementi indiziari elencati al comma 2 dell'art. 630 bis c.p., essendo, per integrare il reato di caporalato, sufficiente la presenza di un solo elemento (così Cass. Sez. IV penale, 2 febbraio 2021, n. 6905). Che per tale reato basti pagare meno il lavoratore v., ad esempio, https://www.studiocataldi.it/articoli/41270-reato-di-caporalato-basta-pagare-meno-il-lavoratore.asp\#ixzz6wiWsurBB [accesso 3 Marzo 2021]. In dottrina cfr., per tutti, L'enigma qualificatorio dei riders. Un incontro ravvicinato tra dottrina e giurisprudenza, in: C. De Marco, A. Garilli, Diritto del mercato del lavoro, Napoli 2021, pp. 1-33. 
Significativa è la restrizione di molti mercati agroalimentari, pesanti sono le ricadute economiche sugli operatori delle filiere, tra i quali i più penalizzati nella distribuzione del valore risultano essere quelli del settore primario ${ }^{2}$.

Nell'anno 2020, in Italia la pandemia ha ridotto le compravendite e ha congelato i prezzi dei terreni agricoli. Sulla base delle statistiche del Consiglio nazionale del notariato per numero di atti, le compravendite di tali beni sono diminuite dell' $8,4 \%$, ribaltando la dinamica positiva in corso dal 2014. Uguale è stato l'andamento dei mutui bancari destinati a finanziare l'acquisto di immobili agricoli che, secondo la Banca d'Italia, hanno bruscamente invertito il trend espansivo protrattosi per quasi un decennio. La carenza di liquidità è stata la causa che più di altre ha contribuito a frenare gli acquisti specialmente nei comparti, poco fa richiamati, che hanno maggiormente subito gli effetti delle restrizioni.

Nel 2021 la progressiva ripresa delle attività sembra cominci a compensare, sia pure parzialmente, la situazione di semiparalisi registrata nell'anno precedente.

\section{Le principali misure finanziarie e giuridiche a sostegno della ripresa dei mercati agroalimentari}

Il work in progress normativo destinato a contenere i contraccolpi dell' emergenza spesso risulta insufficiente e poco efficace. Si richiamano gli interventi europei volti ad adattare programmi e strumenti già esistenti per distillare vini, stoccare carni e prodotti lattiero-caseari, quelli per le deroghe temporanee alle regole sulla concorrenza e per consentire di utilizzare le risorse ancora disponibili nel pilastro dello sviluppo rurale, gli altri sul via libera agli aiuti nazionali nel rispetto del Quadro temporaneo varato nel marzo 2020 dalla Commissione UE per il sostegno dell'economia, accompagnati dall'auspicio, espresso dagli Stati membri - non tutti! - e dall'Europarlamento, verso più adeguate misure finanziarie e di gestione dei mercati.

Nel mese di ottobre 2021 l'esecutivo dell'Unione Europea ha presentato la proposta diretta a prorogare fino al 30 giugno 2022 il predetto regime temporaneo e straordinario per la concessione di aiuti di Stato, mantenendo

${ }^{2}$ Al riguardo non mancano, però, interventi di sostegno. Si ricorda il d.1. n. 73/2021, c.d. "decreto sostegno bis", recante "Contributi a fondo perduto, esoneri e agevolazioni", in cui numerose sono le misure destinate al settore primario: l'esonero dei contributi INPS (Istituto nazionale della previdenza sociale) per il settore vitivinicolo e l'agriturismo, comprese le aziende produttrici di vino e di birra, l'incremento delle percentuali di compensazione IVA per il settore zootecnico, il sostegno all'occupazione per il settore degli agriturismi e le risorse finanziarie destinate al settore dello zucchero, gli interventi a favore dell'imprenditoria femminile. 
invariati i massimali dei sostegni, pari a 225 mila euro per le imprese agricole operanti nella produzione primaria.

Alla data della richiesta di proroga la vice-presidente della Commissione ha osservato che - nonostante ammonti a più di tre miliardi di euro la somma spesa complessivamente dagli Stati membri per limitare le conseguenze economiche della pandemia nel settore agroalimentare e si registri una buona ripresa dell'economia europea - occorre evitare che il ritiro del sostegno pubblico provochi bruschi deterioramenti.

La stessa logica sta alla base del provvedimento con il quale sempre l'Esecutivo UE, nell'agosto 2021, ha consentito agli Stati membri di aumentare, con decorrenza dal 6 ottobre, la percentuale degli anticipi relativi agli aiuti diretti della Pac e alle misure per lo sviluppo rurale. Con un'apposita nota la Commissione ha, infatti spiegato come tale decisione sia finalizzata a "sostenere ed aumentare il flusso di cassa degli agricoltori colpiti dalle conseguenze economiche della pandemia" e dall'aggiunto impatto dovuto alle condizioni meteorologiche avverse - inondazioni ed ondate di calore registrate in numerosi Stati membri.

In Italia la messa in campo di misure straordinarie rivolte a strutture di trasformazione e commercializzazione di prodotti agricoli, nonché a piccole e medie imprese agricole, silvicole e di acquacoltura, in sofferenza di liquidità, benché assistite, quanto all'erogazione di prestiti, da garanzie pubbliche nei limiti degli aiuti di Stato, scontano la scarsa conoscenza in seno al sistema bancario delle peculiarità del comparto agroalimentare e la consueta lentezza burocratica.

Tra gli interventi normativi recanti misure di sostegno economico a famiglie, lavoratori e imprese per attenuare le criticità connesse all'emergenza epidemiologica da COVID-19, il c.d. Decreto Cura Italia ha introdotto l'istituto del pegno rotativo3.

Si tratta di una soluzione di finanziamento innovativa pensata per i produttori DOP e IGP, incluse le aziende della filiera vitivinicola, sotto forma

${ }^{3}$ Si veda il d. 1.17 marzo 2020, n. 18 convertito con modifiche in 1.24 aprile 2020, n. 27. In particolare l'art. 78 concernente le misure in favore del settore agricolo e della pesca, al comma 2-duodecies, prevede che: i prodotti agricoli e alimentari a denominazione d'origine protetta o a indicazione geografica protetta, inclusi i prodotti vitivinicoli e le bevande spiritose, possono essere sottoposti a pegno rotativo, attraverso l'individuazione, anche per mezzo di documenti, dei beni oggetto della garanzia pignoratizia e di quelli sui quali il pegno si trasferisce, nonché mediante l'annotazione in appositi registri. Il comma successivo demanda a un atto ministeriale la definizione di alcuni dettagli ai quali è destinato il decreto 23 luglio 2020, emanato dal Ministero delle politiche agricole alimentari e forestali, contenente la regolazione delle modalità di costituzione, individuazione, annotazione ed estinzione del pegno rotativo su DOP e IGP di prodotti agricoli, alimentari, prodotti vitivinicoli e bevande spiritose. 
di anticipazione su pegno di merce senza spossessamento del bene che resta nei magazzini e nelle cantine dei produttori.

Lo strumento finanziario (peraltro non contingente) è attivabile attraverso l'intervento degli istituti bancari, i quali provvedono alla valorizzazione puntuale dei prodotti e li convertono in garanzie utili per consentire alle imprese di accedere ad ulteriori linee di credito ${ }^{4}$.

Il finanziamento può essere rinnovato automaticamente, sostituendo semplicemente il prodotto dato in pegno con l'altro di nuova produzione. Il pegno consente alle imprese di qualsiasi dimensione di sfruttare gli asset di magazzini e cantine per rinnovare la liquidità utile alla gestione e allo sviluppo dell'attività economica.

Sono specificamente destinate alla ripresa le azioni europee e nazionali che muovono dal Next Generation EU, meglio conosciuto come Recovery Fund, ovvero il Piano da 750 miliardi di euro che dovrà condurre gli Stati membri in una nuova era, posto che, a causa della perdurante emergenza sanitaria scoppiata nel 2020 non sarà possibile tornare al passato, le principali economie di area euro sono cadute in profonda crisi ed è necessario fissare le basi per un futuro in cui la capacità di resilienza risulti rafforzata.

Il nuovo strumento fornisce agli Stati il sostegno finanziario per intensificare gli investimenti pubblici e le riforme dopo la forte contrazione economica avviata dall'emergenza COVID, garantendo al contempo che le loro economie intraprendano le transizioni verde e digitale, diventino più sostenibili e resilienti. Il Recovery Fund assegna ai governi dei singoli Paesi il compito di tradurre i principi in azioni concrete attraverso i rispettivi Piani di rilancio nazionali tenendo bene in conto che Bruxelles non accetterà investimenti dannosi per l'ambientes.

Nel PNRR o $\neq$ Next Generation Italia, l'agricoltura rileva in più parti: nella missione 2 dedicata a Rivoluzione verde e transizione ecologica e, in particolare, nelle linee M2C1 - Economia circolare e agricoltura sostenibi-

${ }^{4} \mathrm{Al}$ riguardo sono state firmate intese con vari gruppi bancari. Si ricorda quella sottoscritta tra Icreea gruppo bancario cooperativo (che conta 130 istituti dislocati sul territorio italiano e vanta nel comparto agroalimentare una quota nazionale del $10 \%$ ), Valoritalia (ente italiano preposto - su incarico del ministero delle Politiche agricole - alla certificazione dei vini DOP e IGP) e Federdoc (che, da oltre 40 anni, rappresenta i consorzi di tutela vitivinicoli, supportando e promuovendo ogni iniziativa tesa a favorire lo sviluppo del settore) per l'utilizzo del pegno rotativo sulle scorte a sostegno del settore del vino, consentendo alle relative aziende di valorizzare tale prodotto in affinamento e convertirlo in garanzie funzionali all'ottenimento di linee di credito dedicate.

${ }^{5}$ Così E. Giovannini, portavoce dell'Alleanza italiana per lo sviluppo sostenibile, nelle dichiarazioni riportate da C. Bussi Recovery Plan, l'occasione per uno scatto di sostenibilità, Il Sole24 Ore 29 settembre 2020, Rapporti. Sviluppo sostenibile, p. 1. 
le, $\mathrm{M} 2 \mathrm{C} 2$ - Energia rinnovabile, ecc., M2C4 - Tutela del territorio e della risorsa idrica; ma il settore primario è implicitamente compreso sia nelle missioni 5 e 6 attinenti rispettivamente ad inclusione sociale e salute, sia nel fondo complementare al PNRR, ove sono previsti anche finanziamenti per i contratti di filiera e di distretto nei settori agroalimentari, ittico, forestale e florovivaistico ${ }^{6}$.

\section{Gli interventi a tutela dei riders del food delivery}

Come si è già accennato nelle prime battute di questo contributo, tra gli effetti della pandemia vi è l'aumento delle esigenze di tutela rivendicate tanto dai lavoratori del settore agricolo quanto da quelli del food delivery.

La tutela della posizione dei prestatori di lavoro nel settore primario rappresenta uno degli aspetti più controversi e dibattuti a livello unionale che ha impegnato i negoziati relativi alla nuova Pac. Il precipuo riferimento alla condizionalità sociale tesa a ridurre gli aiuti in caso di violazione dei diritti dei lavoratori ha acceso una vivace discussione tra $\mathrm{i}$ "co-legislatori e ha visto l'Europarlamento farsi proponente - in contrapposizione a Consiglio e Commissione - di una soluzione di compromesso fondata sulla considerazione dei diritti dei lavoratori e degli agricoltori, con l'intento di incidere il meno possibile sugli oneri burocratici degli Stati.

Quanto alla situazione italiana si richiamano i rimedi messi a punto dalla c.d. legge sul caporalato e quelli in itinere sollecitati dalle azioni giudiziarie promosse dai c.d. riders, i moderni fattorini del food delivery che, provvisti di smartphone, biciclette o motocicli, sfrecciano tra le strade di tanti centri urbani e zone limitrofe per consegnare cibo al domicilio dei consumatori. La tipologia delle loro prestazioni, da qualche tempo già ampiamente diffusa, ha avuto un incremento forte e capillare nei periodi di confinamento dovuti all'emergenza sanitaria, andando ad implementare le situazioni di frizione e conflittualità tra le parti del contratto di lavoro.

Le tensioni tra i riders ed i loro "singolari" datori di lavoro sono dovute alle fattispecie negoziali che regolano le prestazioni, nonché alle concrete

${ }^{6}$ Sul punto è opportuno segnalare come, nell'ottobre 2021, la Commissione europea - in una nota con la quale ha dato il via libera ad un programma spagnolo per l'ammodernamento di serre - abbia precisato che tutti gli investimenti e le riforme comportanti aiuti di Stato inclusi nei piani di ripresa e resilienza degli Stati membri devono essere notificati alla Commissione europea per la preventiva approvazione nel rispetto delle norme in materia di aiuti di stato. Ciò "al fine di preservare la parità di condizioni nel mercato unico, garantendo altresì che le risorse finanziarie siano utilizzate in modo da ridurre al minimo le distorsioni di concorrenza e non escludere gli investimenti privati”. 
modalità di esecuzione delle stesse che, oltre a variare da piattaforma a piattaforma, vengono sottoposte a continui aggiustamenti.

In seguito ad alcuni riconoscimenti provenienti dalla giurisprudenza la questione della tutela dei riders è diventata oggetto di proposte normative, l'ultima delle quali è confluita nel d.1. 3 settembre 2019, n. 101, convertito in 1.2 novembre 2019, n. 128. La novella legislativa estende l'applicazione di talune disposizioni del decreto delegato n. 81/2015 alle modalità di esecuzione delle prestazioni organizzate mediante piattaforme digitali ed aggiunge alla fonte appena citata il Capo V-bis, rubricato Tutela del lavoro tramite piattaforme digitali ${ }^{7}$ e gli articoli dal 47-bis al 47-octies.

Con la novellazione appena richiamata i riders rimangono dei lavoratori autonomi, ma potranno dimostrare in giudizio di essere etero-organizzati in virtù del modificato art. 2 del d.lgs. n. 81/2015 che prescrive "l'applicazione della disciplina del rapporto di lavoro subordinato anche ai rapporti di collaborazione che si concretano in prestazioni di lavoro prevalentemente personali, continuative e le cui modalità di esecuzione sono organizzate dal committente", nonché l'applicazione delle medesime disposizioni anche quando "le modalità di esecuzione della prestazione siano organizzate mediante piattaforme digitali".

Per dare effettività ai diritti riconosciuti dalla legge è stata emanata dal ministero del lavoro la circolare n. 17 del 19 novembre 2020 "Riders - Tutele per i ciclo-fattorini delle piattaforme digitali" e si sono attivati diversi operatori.

I sindacati risultano impegnati nel cercare di estendere al settore le garanzie e le tutele fornite dai contratti nazionali di lavoro; il Ministro del Lavoro in carica, Andrea Orlando, ha posto, nella primavera del 2021, il tema dei riders e dei nuovi lavori al centro della propria azione; non sono mancate alcune iniziative di europarlamentari volte a sollecitare la proposta da parte della Commissione Europea di una direttiva ad hoc per i lavoratori delle piattaforme digitali.

Contemporaneamente sono state adottate talune azioni concrete. Il riferimento va alla l.r. Toscana approvata nel maggio 2021 e al modello contrattuale predisposto da Just Eat, la nota app leader per ordinare online, e in tutto il mondo, la consegna di pranzo e cena a domicilio.

$\mathrm{Nel}$ rispetto delle competenze regionali in materia, il primo intervento intende garantire più mezzi a tutela della salute e della sicurezza dei riders e degli altri lavoratori delle piattaforme digitali: valutazione dell'esposizione

${ }^{7}$ Il d.lgs. 15 giugno 2015, n. 81, più volte modificato, contiene la "Disciplina organica dei contratti di lavoro e revisione della normativa in tema di mansioni, a norma dell'articolo 1 , comma 7 , della legge 10 dicembre 2014, n. 183". 
ai rischi, visite mediche preventive, formazione specifica per il "lavoro sulla strada", fornitura di adeguati strumenti di protezione individuale ${ }^{8}$.

Con l'anno 2021 Just Eat ha introdotto in Italia (ove è presente dal 2011) il modello di consegna a domicilio Scoober, già attivato dal medesimo gruppo in altri Paesi europei. Lo schema negoziale inquadra i riders come lavoratori dipendenti e, in quanto tali, completamente destinatari delle pertinenti tutele e assicurazioni. Il prototipo prevede la stipula di un contratto in cui i riders vengono qualificati dalla piattaforma danese come lavoratori dipendenti e ciò seguendo le linee guida internazionali predisposte in uno schema di accordo aziendale opportunamente conformato alla normativa e alla legislazione italiana.

\section{Le altre cause incidenti sulla potenzialità produttiva delle filiere agroalimentari. Il proliferare di Accordi preferenziali sul mercato internazionale}

Contemporaneamente alle fragilità provocate dalla crisi sanitaria, l'agricoltura e i settori ad essa collegati incontrano le incertezze sulle potenzialità produttive determinate dal cambiamento climatico, dalla crescita della popolazione mondiale e dalle connesse esigenze di food security, dall'aumento degli Accordi preferenziali sul commercio internazionale di prodotti agroalimentari e delle dinamiche concorrenziali a scapito del relativo comparto dell'Unione.

In proposito è opportuno ricordare che dal $1^{\circ}$ gennaio 2021, a seguito del definito recesso dall'UE, il Regno Unito ha riassunto la piena potestà di sottoscrivere accordi commerciali bilaterali con i Paesi terzi e ha raggiunto il nuovo accordo di libero scambio post Brexit con l'Australia, anche se restano da superare alcuni ostacoli, inclusi quelli relativi al capitolo agricolo, rispetto al quale la NFU - principale organizzazione degli agricoltori del Regno Unito - ha manifestato una forte cautela riservandosi di esaminare

${ }^{8}$ Da almeno 5 anni la regione Toscana ha predisposto una cornice legislativa sulle regole da rispettare in coerenza con le quali sono stati costruiti accordi specifici di settore con le parti sociali: dal marmo alla carta, dal legno alla chimica e all'agricoltura. La fonte, approvata dal Consiglio Regionale il 25 maggio 2021, prevede che anche il settore delle "piattaforme digitali" rientri in questa azione di prevenzione e controllo mirato da parte delle Unità sanitarie locali. La novella normativa rappresenta il primo intervento regionale di questo tipo e ha pertanto aperto l'auspicio che sia "esportato" in tutte le altre Regioni per garantire un maggiore controllo, una maggiore sicurezza e le doverose tutele ad una categoria di lavoratori che di diritti riconosciuti ne ha davvero pochi: queste in sintesi sono alcune delle dichiarazioni espresse, subito dopo l'approvazione, da alcuni esponenti dell'organo regionale. 
nel dettaglio le disposizioni sulla protezione dell'ambiente e sul benessere degli animali allo scopo di verificare se gli elevati standard di produzione adottati nel Paese non siano compromessi dai termini dell'accordo.

In Spagna, l'Asaja - associazione giovani agricoltori - ha denunciato la necessità di reagire alla crescente concorrenza sud africana e richiede di rivedere la vigente intesa di cooperazione tra l'UE e il Sudafrica a fronte dell'andamento delle esportazioni degli agrumi sul mercato dell'Unione in aumento del $30 \%$ nel 2020. In senso contrario, e dunque con il sollievo di molti esportatori agroalimentari europei tra cui quelli italiani, è diretto l'accordo siglato a Bruxelles, anch'esso bilaterale, USA-UE con il quale, nel giugno 2021, i presidenti Biden e Von der Leyen hanno deciso di prolungare per cinque anni la tregua raggiunta nel precedente mese di marzo sui dazi aggiuntivi statunitensi e hanno così sospeso le misure di ritorsione in atto tra le due sponde dell'Atlantico.

L'intesa è considerata dai Paesi europei molto importante sia in considerazione dell'elevato prezzo pagato alla pandemia dal settore agroalimentare dell'Unione a causa della lunga chiusura di bar e ristoranti in tutto il mondo", sia perché dà l'avvio a un dialogo costruttivo e all'adozione di un metodo di soluzione delle dispute commerciali che dovrebbe contribuire al rilancio del sistema multilaterale di gestione degli scambi commerciali a livello globale.

\section{5. (Segue). Il Green Deal, le incertezze del clima, la delicata posizione del comparto agroalimentare. Le osservazioni e le reazioni di importanti attori istituzionali. Le preoccupazioni e le reazioni degli operatori e dei consumatori}

A fronte dello scenario globale sinteticamente delineato il ruolo strategico del settore primario e del sistema agroalimentare, richiederebbe alle Istituzioni europee e nazionali di agire con una visione d'insieme attraverso una programmazione coordinata e non di breve termine.

Negli ultimi mesi si sono susseguite le preoccupazioni relative al differimento della riforma della politica agricola comune, alle difficoltà dei relativi negoziati, alle questioni del budget, alle intemperanze climatiche, alle strategie di biodiversità e Farm to Fork proposte dalla Commissione -

${ }^{9}$ Si pensi che, quanto al Made in Italy, gli Stati Uniti rappresentano per l'agroalimentare il primo mercato di sbocco fuori dai confini comunitari, con un valore, secondo elaborazioni Istat, di 4,9 miliardi in cibi e bevande nel 2020 ma in calo del 2\% nel primo trimestre del 2021. 
che costituiscono la parte agricola del Green Deal europeo - alle difficoltà incontrate dalle amministrazioni nazionali nella fase di stesura dei piani nazionali, una delle maggiori novità contenute nella riforma della Pac varata nel giugno $2021^{10}$.

$\mathrm{Al}$ contempo richiederebbero una valutazione il più possibile oggettiva le segnalazioni e i rammarichi provenienti da alcuni organi istituzionali dell'Unione e da talune organizzazioni internazionali, laddove denunciano la perdurante scarsa efficacia dei finanziamenti e delle misure della Pac per dare risposta all'esigenza di riduzione delle emissioni e delle pratiche agricole irrispettose dell'ambiente.

Il 9 giugno 2021 il Parlamento dell'Unione ha approvato con un'ampia maggioranza la risoluzione recante la "Strategia sulla biodiversità per il 2030 - Riportare la natura nella nostra vita", in cui viene affrontato l'attuale stato di crisi della biodiversità in Europa e nel resto del mondo.

I deputati hanno accolto favorevolmente le ambizioni di ripristinare, rendere resilienti e proteggere adeguatamente gli ecosistemi entro il 2050, hanno manifestato il rammarico per il mancato raggiungimento nell'Unione europea degli obiettivi per la biodiversità fissati al 2020 e ritengono che la nuova strategia debba affrontare in modo efficace le cinque principali cause dei mutamenti: i cambiamenti nell'uso del suolo e del mare, lo sfruttamento diretto degli organismi, i cambiamenti climatici, l'inquinamento, le specie esotiche invasive.

Infine, gli eurodeputati hanno quantificato la necessità di assegnare $20 \mathrm{mi}-$ liardi di euro all'anno per favorire la biodiversità nel territorio europeo e hanno auspicato la stipula di un "accordo di Parigi" sulla biodiversità in seno alla Conferenza delle Nazioni Unite tenuta in Cina nell'ottobre 2021 con l'obiettivo di stabilire le priorità globali in materia di biodiversità per il 2030 e oltre.

Sempre nel medesimo mese di ottobre, è stata approvata dal Parlamento dell’UE la relazione sulla comunicazione della Commissione Farm to Fork

${ }^{10}$ Le tante novità rispetto alla normativa vigente e la pubblicazione dei regolamenti di base prevista non prima degli ultimi giorni di novembre rendono difficili la stesura dei piani nazionali e il loro invio a Bruxelles entro la scadenza fissata a fine dicembre del 2021. Agli inizi di ottobre i lavori erano ancora in corso in Francia e Spagna, in Germania, l'esito delle elezioni politiche lasciava intravedere una revisione delle scelte fatte dal Parlamento uscente, in Italia erano state svolte solo due riunioni del Tavolo di partenariato - costituito dal ministero dell'Agricoltura - dedicate agli ecoschemi, vale a dire la parte degli aiuti diretti della nuova Pac condizionati alla messa in opera, da parte degli agricoltori e su base volontaria, di pratiche colturali con una maggiore valenza ambientale (le notizie sono tratte da Agrisole online del 7 ottobre 2021). Sempre ad ottobre il Consiglio ha deciso di negare il rinvio, richiesto da alcuni Stati, della data per la presentazione dei piani strategici, ma, contestualmente, ha invitato la Commissione ad adottare "un approccio trasparente nella valutazione dei piani fornendo chiari criteri di valutazione". 
nella quale è contenuta la richiesta di fissare i target vincolanti per la riduzione dei prodotti chimici utilizzati in agricoltura. Inoltre, l'Europarlamento ha sollecitato sia la revisione delle norme sulla concorrenza allo scopo di rafforzare il ruolo degli agricoltori all'interno della filiera agroalimentare, sia l'adozione di misure ad hoc per ridurre i consumi di carne e la progressiva eliminazione degli allevamenti in gabbia, sia una maggiore attenzione alle tecniche innovative, comprese quelle genomiche.

Nella specifica relazione, EN 2021,16, la Corte dei Conti dell'UE ha "bocciato" le misure ambientali della Pac 2013-2020, rilevando come i finanziamenti europei, oltre a non avere contribuito a ridurre le emissioni di gas a effetto serra prodotte dall'agricoltura, hanno addirittura incentivato pratiche non rispettose dell'ambiente. Dopo aver constatato il fallimento del greening, i giudici hanno evidenziato la necessità di cambiare passo già nell'ambito della riforma in itinere. A tale proposito il giudice relatore ha dichiarato che: "La UE svolge un ruolo fondamentale nella mitigazione dei cambiamenti climatici nel settore agricolo, dal momento che elabora normative in materia di ambiente e cofinanzia la maggior parte della spesa agricola degli Stati membri"; "la nuova politica agricola comune deve concentrarsi di più sulla riduzione delle emissioni prodotte dall'agricoltura, deve essere più trasparente e rendere meglio conto del contributo fornito alla mitigazione dei cambiamenti climatici".

Lo stesso Collegio giudicante ha bocciato le misure della Pac relative alla gestione sostenibile delle risorse idriche in agricoltura, ritenendole non idonee a far sì che gli agricoltori utilizzino l'acqua in modo sostenibile.

La Corte dei Conti ritiene che "nonostante il settore primario incida sulle risorse idriche in misura marcata, le troppe esenzioni - rispetto agli obblighi stabiliti dalla politica dell'Unione in materia di acque - riconosciute agli agricoltori ostacolino gli sforzi diretti ad assicurare un utilizzo idrico sostenibile e che la Pac spesso finisca per promuovere o sostenere un maggiore impiego dell'acqua, anziché incentivarne una maggiore efficienza".

Anche in sede di WTO il settore agricolo riceve critiche del medesimo tenore. Nell'ottobre 2021, durante lo svolgimento a Washington degli incontri tra i vertici della Banca mondiale del commercio, la direttrice generale dell'Organizzazione mondiale del commercio, ha evidenziato la necessità di raggiungere un accordo per porre fine ai sussidi all'agricoltura in quanto creano uno svantaggio per i Paesi meno avanzati e danneggiano l'ambiente.

Qualche settimana prima è stata avviata un'azione per raggiungere un'intesa multilaterale sulle questioni agricole durante la Conferenza ministeriale della WTO programmata per la fine di novembre. Nell' ambito del documento (non reso pubblico), che è circolato tra i rappresentanti dei Paesi aderenti alla 
WTO, le indiscrezioni diffuse riferiscono circa la formulazione di proposte concernenti i limiti al sostegno interno agli agricoltori, la costituzione di stock pubblici ai fini della sicurezza alimentare, le modalità di attivazione, da parte dei singoli Paesi, della clausola di salvaguardia in caso di eccessivo aumento delle esportazioni e delle conseguenti pressioni al ribasso sui prezzi interni ${ }^{11}$.

Altrettanto severa è la valutazione formulata dall'OCSE nel rapporto riferito al periodo 2018-2020, in cui l'Organizzazione internazionale per la cooperazione e lo sviluppo economico pone in evidenza che, nell'arco temporale considerato, oltre un terzo dei trasferimenti erogati annualmente al settore agricolo è stato destinato al sostegno delle quotazioni, con il risultato di fare pagare prezzi alti ai consumatori, mentre alle innovazioni è andato soltanto il $6 \%$.

In ragione di questi dati l'OCSE ha sottolineato l'inefficacia degli interventi sui prezzi di mercato e di limitazione del commercio, anche perché espongono a rischio la sicurezza alimentare su scala mondiale e ostacolano il collocamento dei prodotti alimentari dalle regioni eccedentarie a quelle deficitarie, altresì determinando la volatilità dei prezzi sui mercati internazionali.

Ad avviso dell'Organizzazione ciò rende indispensabile un profondo cambiamento degli obiettivi e delle modalità di attuazione delle politiche agricole, al centro delle quali, una volta superata l'emergenza sanitaria, le autorità pubbliche dovranno porre le iniziative di sostegno all'innovazione accompagnandole con la soppressione progressiva del sistema degli interventi sui prezzi e il sostegno diretto all'agricoltore ${ }^{12}$.

D'altro canto, meritano altrettanta attenzione le preoccupazioni e i rilievi mossi dagli operatori del settore primario e delle filiere connesse (alimentare e industriale), nonché dalle associazioni di consumatori ed utenti in ordine all'impatto del Green Deal in termini di riduzione intorno al 20\% delle produzioni, di aumento dei prezzi al consumo stimati fino al 50\%.

In merito può assumere un certo interesse la valutazione di due professori dell'Università tedesca di Kiel riferita al documento della Commissione UE e pubblicata nel settembre 2021.

Sul fronte dell'impatto climatico gli studiosi stimano una riduzione, a livello europeo, delle emissioni di gas serra nella misura del $30 \%$ rispetto alla situazione in atto, ma osservano come la riduzione sarebbe però annullata dal minore trattenimento nel suolo del carbonio dovuto alla contrazione delle produzioni. Per di più aumenterebbero le emissioni nei Paesi terzi, che

${ }^{11}$ Le notizie sono contenute nell'articolo WTO, proposta per azzerare i sussidi agricoli che danneggiano ambiente e PMA, Agrisole online, 22 ottobre 2021.

${ }_{12}$ Cfr., al riguardo, Sussidi agricoli oltre quota 600 miliardi ma con pochi benefici per ambiente e sicurezza, Agrisole online, 24 giugno 2021. 
dovrebbero accrescere le loro esportazioni agroalimentari verso gli Stati membri per soddisfare il bisogno alimentare dei cittadini europei. Dunque, il bilancio sarebbe a somma zero, se non addirittura negativo.

Inoltre, non possono essere ignorate le osservazioni critiche al Green Deal europeo formulate dagli USA, già nella riunione fiorentina del 1718 settembre 2021, dai Ministri dell'Agricoltura del G-20 e poi confermate, qualche giorno dopo, al vertice ONU di New York sui sistemi alimentari.

Secondo il segretario di Stato americano, Tom Vilsack, le linee proposte dall'esecutivo UE non rappresentano la soluzione per contrastare il cambiamento climatico, in quanto, sulla base di uno studio redatto dal dipartimento di Stato USA, se applicate a livello mondiale, le indicazioni contenute nella comunicazione dal "Produttore al consumatore" e la nuova strategia europea per la biodiversità farebbero diminuire di oltre il $10 \%$ la produzione agricola, nonché del $17 \%$ i redditi degli agricoltori oltre a determinare un aumento dei prezzi che potrebbero arrivare quasi al 90\%. Per raggiungere la neutralità climatica dell'agricoltura e preservare l'efficienza delle imprese andrebbero potenziati l'uso delle nuove tecniche genomiche, dell'agricoltura di precisione e dell'intelligenza artificiale.

Considerazioni simili sono riscontrabili nella recente relazione redatta dal Centro comune di ricerca $(\mathrm{Ccr})$, che fornisce consulenze scientifiche alla Commissione UE.

Infine, occorre ricordare come, tra le diffuse preoccupazioni, che richiedono di essere contenute attraverso adeguate azioni e misure pubbliche e private, vi siano quelle derivanti dall'impatto negativo sulla produttività del settore agroalimentare delle calamità naturali determinate dalle schizofreniche intemperanze climatiche (alluvioni, gelate e grandine, siccità, desertificazione, incendi) ${ }^{13}$.

Alle valutazioni provenienti dai vari contesti appena richiamati, la Commissione ha reagito ufficialmente non contestandole, ma mettendone in

${ }^{13}$ Non mancano le misure rimediali, tra le quali vi sono: gli strumenti anche finanziari per rafforzare le polizze assicurative contro la perdita delle produzioni, quelli destinati a prevenire e contrastare gli incendi e a sviluppare nuovi processi e tecnologie per accrescere la competitività e la sostenibilità del settore primario, i piani adottati da imprese private, quale, ad esempio, la strategia Eating for the Planet messa a punto da Nomad Foods, il più grande produttore di surgelati in Europa, cui partecipa Findus Italia, per ridurre significativamente le riduzioni del global warming. Oltre a ridurre del $25 \%$, nel prossimo quadriennio, le emissioni direttamente o indirettamente prodotte attraverso l'acquisto di beni, trasporto e distribuzione, Nomad Foods si impegna a fornire un supporto ai propri fornitori, assicurando che il $75 \%$ di quelli più inquinanti sviluppi gli obiettivi di riduzione delle emissioni - basati su criteri scientifici - entro il 2025. Per ulteriori dettagli dell'iniziativa si demanda a Nomad Foods, per i fornitori inquinanti giro di vite sulle emissioni entro il 2025, Agrisole online, 10 settembre 2021. 
evidenza la parzialità dovuta alla mancanza o alla scarsa considerazione di alcuni aspetti quali il cambiamento delle scelte dei consumatori e l'impatto delle innovazioni tecnologiche. Contemporaneamente l'Esecutivo UE ha diffuso una serie di dati dai quali risulta come la siccità provochi ormai danni in agricoltura di circa nove miliardi di euro all'anno e l'aumento della temperatura sia in grado di causare una contrazione fino al $10 \%$ delle rese di grano, mais e riso ${ }^{14}$.

\section{Qualche riflessione conclusiva}

Le istanze, le valutazioni e i rammarichi, uniti alla preoccupante posizione espressa dalla Corte dei Conti europea, inducono a sollecitare i centri di potere competenti e gli stakeholders economico-sociali a svolgere in tempi rapidi un'adeguata e profonda riflessione sul futuro della sovranità alimentare in Europa e sulle funzioni della Pac.

Né si può trascurare la questione dell'accesso al cibo a livello globale.

In merito si segnalano le notizie diffuse dalla FAO nel giugno 2021 attestanti l'aumento record dei prezzi per i generi alimentari registrati nel maggio precedente (al ritmo mensile più rapido dell'ultimo decennio) e ciò, nonostante, la produzione mondiale di cereali fosse in procinto di segnare un nuovo record.

Nell'intervento effettuato al pre-vertice delle Nazioni Unite sui Sistemi alimentari, ospitato dal governo italiano nel luglio 2021, il Presidente del Consiglio Mario Draghi ha ricordato le numerose minacce alla food security e ha promosso una coalizione alimentare, a cui hanno aderito 40 Paesi, con l'obiettivo di raggiungere la "Sicurezza alimentare per tutti", combattendo la povertà estrema e l'insicurezza alimentare incrementata dalla pandemia.

Recentemente - alla vigilia del vertice internazionale di Glasgow per i negoziati sul clima della COP26 - un gruppo composto da venti banche pubbliche di sviluppo (PDBs) ha annunciato un'iniziativa volta ad accelerare gli investimenti eco in agricoltura con l'intento di aumentare i finanziamenti destinati agli agricoltori più poveri del mondo e più colpiti dal cambiamento climatico.

L'iniziativa consisterà nella messa a punto di una piattaforma volta ad intensificare investimenti ecologici ed inclusivi in agricoltura nonché in

${ }^{14}$ La Commissione ha inoltre evidenziato che l'agricoltura europea è stata in grado nel decennio scorso di ridurre l'uso dei prodotti chimici e le emissioni di gas ad effetto serra (meno $25 \%$ sui livelli del 1990), aumentando nello stesso tempo la produzione e le esportazioni almeno del $10 \%$ all'anno dal 2010 al 2020. 
attività di elaborazione, confezionamento e trasporto degli alimenti nel loro percorso dalla fattoria al piatto. La piattaforma fornirà servizi di assistenza tecnica, condivisione di competenze e strumenti per quantificare l'impatto sociale ed ambientale degli investimenti e realizzare una migliore valutazione dei rischi.

La proposta è risultata in linea con alcune delle osservazioni espresse durante lo svolgimento della COP 26 secondo le quali la salute del Pianeta richiederebbe soluzioni su vasta scala, possibili da attuare soltanto se si costituiranno partnership abbastanza strette tra i governi, le principali banche multilaterali, il settore privato e i suoi investitori ${ }^{15}$.

Per conto dell'ONU il Segretario generale Guterres ha posto l'accento sulla necessità di azioni globali per realizzare sistemi alimentari più inclusivi e sostenibili sottolineando come i sistemi alimentari abbiano un grande impatto sulle nostre economie, sulla nostra salute e sull'ambiente in quanto rappresentano uno dei principali motivi per i quali non riusciamo a restare entro i confini ecologici del pianeta. Al contempo - ha proseguito Guterres i medesimi sistemi possono essere la chiave per contrastare la crisi climatica, la crescente riduzione della biodiversità e per costruire società più sane ${ }^{16}$.

Se a queste considerazioni si aggiungono sia le deludenti posizioni assunte al vertice di Glasgow da alcuni dei Paesi grandi inquinatori in ordine al taglio delle emissioni di gas serra per contenere il global warming, sia l'intesa raggiunta - comunque al ribasso (si pensi che, invece, di stabilire l'eliminazione si raccomanda la riduzione progressiva delle emissioni di $\mathrm{CO}_{2}$ ) - trasfusa nel documento conclusivo dei difficili negoziati ${ }^{17}$, sono inevitabili le riflessioni che inducono a formulare la seguente esortazione, riferibile innanzitutto al contesto europeo: "va bene la forte riduzione di pesticidi e fertilizzanti, ma attenzione all'introduzione di vincoli - come le riserve predeterminate di superfici agricole per produzioni biologiche o per trasformarle in aree ad alta biodiversità - che

${ }^{15}$ Tra tutti si richiama l'intervento di Sua Altezza reale Principe Carlo, Principe di Galles.

${ }^{16}$ Per maggiori dettagli si rinvia agli articoli $F A O$, prezzi record per i prodotti alimentari: è il valore più alto dal settembre 2011, Agrisole online, 3 giugno 2021 e Draghi: agire subito per contrastare la crisi alimentare, più fondi da governi e banche, Agrisole online, 26 luglio 2021.

17 Com'è noto i giudizi sull'intesa raggiunta a conclusione della COP 26, pur essendo di matrice diversa, esprimono una diffusa presa d'atto di risultati inferiori alle aspettative. Le qualificazioni oscillano tra "compromesso", "miglior accordo possibile", passo avanti", "troppo poco". I principali punti dell'intesa sono stati così sintetizzati: global warming (soglia critica a 1,5 gradi); emissioni $\mathrm{CO} 2$ (zero netto intorno a metà secolo); carbone e sussidi (riduzione graduale); rammarico per il minor importo (rispetto agli impegni assunti nel 2009) versato ai Paesi a basso reddito da parte di quelli ad economie avanzate, (queste le prime notizie riferite da G. Di Donfrancesco, Cop26, intesa che salva gli impegni presi a Parigi, Il Sole 24 Ore, 14 novembre 2021, p. 7, giorno in cui è stato concluso il presente contributo. 
possono frenare e discriminare gli input produttivi degli agricoltori, incidendo sulla sovranità alimentare dell'Unione".

In definitiva, ciò sta a significare che non può essere il comparto agroalimentare la "vittima" principale da sacrificare alla causa del climate chance. Per il settore primario la "Pac deve restare una politica economica, e, per questo, dev'essere snella, flessibile, in grado di favorire lo sviluppo di tutte le imprese che producono per il mercato e assicurano occupazione": questo è il condivisibile messaggio diffuso dai vertici di tre organizzazioni agricole di Italia, Germania, Francia ${ }^{18}$, nei giorni immediatamente precedenti alla riunione dei ministri dell'Agricoltura europei, calendarizzata dalla Presidenza del Consiglio nel maggio 2021 in contemporanea con il "Trilogo" istituzionale Commissione - Consiglio - Parlamento, nel quale si auspicava il raggiungimento dell'accordo sulla riforma della Pac post 2022, accordo poi siglato il successivo 25 giugno.

Tradotto in termini più strettamente giuridici il messaggio è in linea con i rilievi degli studiosi del diritto che sottolineano la necessità di considerare come il pieno rispetto delle norme europee di rango costituzionale, pressochè invariate, richieda l'impiego di azioni integrate in cui le politiche agricole, con gli opportuni correttivi ecologici, favoriscano produzioni agroalimentari sostenibili anche sotto i profili economico e sociale, affinché con esse si possa competere sui mercati locali e globali garantendo una maggiore difesa della sovranità del cibo, tenendo in debito conto le minacce derivanti dalle intemperanze climatiche ${ }^{19}$.

\section{BIBLIOGRAFIA}

Bussi C. (2020), Recovery Plan, l'occasione per uno scatto di sostenibilità, Il Sole24 Ore 29 settembre 2020, Rapporti. Sviluppo sostenibile.

D’Addezio M. (2020), Mobilità, flessibilità, lettura sistemica dei principi rilevanti: il file rouge per riflettere intorno al rapporto tra agricoltura e costituzione, "Rivista di diritto agrario" n. 1.

De Marco C., Garilli A. (2021), Diritto del mercato del lavoro, Napoli.

Di Donfrancesco G. (2021), Cop26, intesa che salva gli impegni presi a Parigi, Il Sole 24 Ore, 14 novembre.

${ }^{18}$ Si tratta delle organizzazioni agricole Confagricoltura (Italia), Fnsea (Francia) e Dvb (Germania).

${ }^{19}$ Al riguardo si demanda alle più articolate considerazioni svolte in: M. D'Addezio, Mobilità, flessibilità, lettura sistemica dei principi rilevanti: il file rouge per riflettere intorno al rapporto tra agricoltura e costituzione, "Rivista di diritto agrario" 2020, n. 1, p. 553, specialmente le p. 565. 


\title{
AGRI-FOOD SUPPLY CHAINS DURING THE COVID-19 PANDEMIC IN EUROPE AND ITALY. \\ A CHALLENGE BETWEEN FOOD SECURITY, RECOVERY \\ AND MARKET DINAMICS, LABOUR AND CONSUMER PROTECTION, EUROPEAN GREEN DEAL AND CLIMATE UNCERTAINTY
}

\begin{abstract}
Summary
The article examines, first of all, the regulations that the European Union and Italy have adopted to support agri-food supply chains and market recovery after the downturn caused by the COVID-19 pandemic. The author then focuses on the delicate relationship between the agri-food sector and the climate change and its impact on the agri-food sector, as well as on the other causes that are currently threatening its productive capacity. The need for its sustainable ecological development, as well as for greater protection of food sovereignty is also stressed.
\end{abstract}

Keywords: agri-food chains, COVID-19, climate change, food sovereignty

\section{LE FILIERE AGROALIMENTARI AL TEMPO DEL COVID-19 IN EUROPA E IN ITALIA. \\ UNA SFIDA TRA FOOD SECURITY, RIPRESA E DINAMICHE DEI MERCATI, TUTELA DEL LAVORO E DEI CONSUMATORI, GREEN DEAL, INCERTEZZE CLIMATICHE}

\section{Riassunto}

Il saggio, dopo aver considerato alcuni provvedimenti normativi adottati dall'UE e dall'Italia per sostenere le filiere agroalimentari e la ripresa dei relativi mercati a seguito delle contrazioni determinate dalla pandemia COVID-19, si sofferma sul delicato rapporto tra il comparto agroalimentare e il cambiamento climatico e sulle ulteriori cause che attualmente minacciano le potenzialità produttive del comparto, segnalando la necessità di uno sviluppo ecosostenibile di esso ma anche l'esigenza di assicurare una maggiore difesa della sovranità del cibo.

Parole chiave: filiere agroalimentari, COVID-19, cambiamento climatico, sovranità del cibo 